\title{
TOXICIDADE DA AZATIOPRINA NA DOENÇA DE CROHN: INCIDÊNCIA, ABORDAGEM E EVOLUÇÃO
}

\author{
Márcia Valéria Colli*, Thalita Amaral Amaro, André luís Tavares Pinto, Pedro Duarte Gaburri, Júlio Maria Fonseca Chebli \\ Trabalho realizado no Centro de Doenças Inflamatórias Intestinais do Hospital Universitário da Universidade Federal de Juiz de Fora, Juiz de Fora, MG.
}

*Correspondência:

Rua Cel. Júlio Soares, 343

Ubá - MG

CEP 36500-000

Tel. (32) 3531-5574

marciavcolli@yahoo.com.br

\section{RESUMO}

OBjetivo. A azatioprina (AZA) tem sido usada freqüentemente no tratamento da doença de Crohn (DC). O objetivo do presente estudo foi avaliar a freqüência, evolução e abordagem dos efeitos adversos da AZA no tratamento de pacientes com DC.

Métodos. Foram incluídos prospectivamente 106 pacientes portadores de DC em uso de AZA, de janeiro de 2002 a dezembro de 2006. Registraram-se dados clínicos e demográficos, com controle laboratorial mensal dos efeitos hematológicos e supervisão de reações adversas por meio de avaliação clínica. Realizou-se comparação entre os grupos com e sem efeitos adversos.

Resultados. Cinqüenta e seis (52,7\%) dos pacientes estudados apresentaram pelo menos um efeito adverso, requerendo redução transitória da dose da droga; 18 (17\%) necessitaram suspender definitivamente o uso de AZA, geralmente devido a reações de hipersensibilidade. Náuseas e vômitos, freqüentemente leves, ocorreram em 29 (27,4\%); a raça negra e aqueles com comorbidades apresentaram mais intolerância gástrica do que os brancos e aqueles sem outras doenças associadas $(p=0,04)$. Leucopenia foi o efeito adverso mais freqüente, ocorrendo em $36(34 \%)$. 0 tempo de uso de AZA foi maior em pacientes com leucopenia do que nos não leucopênicos $(p=0,00 \mathrm{I})$, enquanto a dose média de AZA foi menor naqueles com leucopenia comparados aos não leucopênicos $(p=0,005)$. Não houve infecções graves, neoplasias ou óbitos durante 0 tratamento com AZA.

Conclusão. A AZA mostrou ser uma droga relativamente segura no tratamento da DC, desde que seja mantida supervisão clínica e laboratorial periódica durante todo o tratamento.

Unitermos: Doença de Crohn. Azatioprina. Toxicidade.

\section{INTRODUÇÃo}

A doença de Crohn (DC) é uma doença inflamatória intestinal (DII) de curso crônico, recorrente e imprevisível, que pode afetar qualquer segmento do trato digestivo'. São comuns as manifestações extraintestinais, as quais podem envolver vários sistemas orgânicos ${ }^{2}$. Vários estudos demonstraram que houve grande aumento mundial na incidência da DC ao longo da segunda metade do século $X X^{3,4}$, o que também ocorreu no Brasil2,5. A terapêutica medicamentosa, antes limitada aos corticosteróides, antibióticos e aminossalicilatos, inclui hoje os imunossupressores/imunomoduladores e terapêuticas biológicas, sendo essas duas últimas classes empregadas cada vez mais freqüentemente entre nós, e muitas vezes capazes de recuperar pacientes antes considerados intratáveis ${ }^{6}$.

Dentre os imunomoduladores, destacam-se os análogos da purina: a 6-mercaptopurina (6-MP) e seu precursor, a azatioprina (AZA)7. As principais indicações para o tratamento com estas drogas incluem: dependência ou refratariedade aos esteróides; tratamento da doença fistulizante; e, possivelmente, prevenção de recorrência pós-operatória ${ }^{8}$. Ambos os agentes podem ser usados nestes casos com a escolha geralmente baseada na experiência prévia do clínico. Entretanto, o uso da AZA/6-MP pode apresentar várias reações adversas, algumas idiossincrásicas e outras dose-dependentes, as quais podem limitar o seu uso mais amplo?. Ao prescrever estas drogas, portanto, é imperioso compreender sua potencial toxicidade e monitorar adequadamente os pacientes para garantir sua segurança e eficácia ${ }^{10}$.

Recentemente, um estudo realizado em nosso meio, avaliando os resultados no longo prazo do tratamento da $D C$ com $A Z A^{\prime \prime}$, porém enfocando especificamente o grupo de pacientes córtico-dependentes, observou a relativa segurança da droga neste subgrupo de pacientes. Não obstante, poucos dados na literatura nacional são disponíveis a respeito da segurança da AZA no tratamento da DC, particularmente em estudos prospectivos longitudinais. Assim, os objetivos do presente estudo foram avaliar a incidência, evolução e abordagem dos efeitos adversos da AZA em pacientes portadores da DC.

\section{Métodos}

Foram incluídos todos os pacientes com DC em uso de AZA acompanhados no Centro de Doenças Inflamatórias Intestinais do Serviço de Gastroenterologia do Hospital Universitário - Universidade Federal de Juiz de Fora, MG, durante o período de janeiro de 2002 a dezembro 
de 2006. A indicação de AZA nos pacientes com DC incluiu: tratamento de doença moderada a grave em pacientes dependentes ou refratários aos esteróides; presença de fístulas; profilaxia de recorrência pós-operatória. Os pacientes incluídos no estudo poderiam estar usando concomitantemente corticosteróides, aminossalicilatos e/ou antibióticos direcionados à DC. Foram excluídos pacientes que apresentaram quaisquer dos seguintes eventos: complicação séptica atual; doença fulminante requerendo hospitalização ou cirurgia imediata; história atual ou pregressa de qualquer tipo de malignidade; história atual ou pregressa de displasia colônica; infecções ativas; infecções graves nos últimos três meses precedendo o início do estudo; pacientes com significantes comorbidades clínicas. Gestantes ou lactantes também foram excluídas, e mulheres em idade fértil foram orientadas a utilizar métodos contraceptivos eficazes. Além disso, foram excluídos os pacientes recebendo tratamento concomitante com infliximab ou outra terapia biológica, talidomida, tacrolimus, micofenolato de mofetil, ciclosporina, methotrexate ou qualquer outra droga em investigação.

Todas as reações adversas atribuídas à AZA foram registradas, abordadas e acompanhadas evolutivamente. $\mathrm{Na}$ inclusão ao estudo, foram anotados os dados relativos aos critérios de elegibilidade, demográficos, história clínica e medicações de uso habitual. Assim, registrou-se o sexo, raça e idade dos pacientes; duração, localização e fenótipo da DC; dose e tempo de uso da AZA; uso de drogas concomitantes direcionadas à doença. Utilizou-se a classificação de Viena ${ }^{12}$ para a determinação da localização e do fenótipo da DC. Antes do início do tratamento com AZA, rotineiramente, foram obtidas amostras de sangue para avaliação hematológica e bioquímica. Os pacientes foram acompanhados nas visitas ambulatoriais em intervalos de um a dois meses, dependendo do julgamento clínico em cada caso individual. Nestas ocasiões, possíveis efeitos adversos relatados pelos pacientes, espontaneamente ou no interrogatório direto, foram devidamente registrados. Todos os pacientes foram submetidos a rotinas laboratoriais para monitoramento da toxicidade durante todo o tratamento. Dessa forma, foram realizados hemograma, contagem plaquetária e dosagem dos níveis séricos de amilase e aminotransferases à admissão ao estudo. O monitoramento subseqüente envolveu hemograma a cada duas semanas, durante oito semanas; depois, a cada um ou dois meses durante o período da terapia. A dosagem de aminotransferases (ALT e AST) foi realizada em intervalos de três meses indefinidamente. Níveis séricos de amilase foram avaliados apenas durante os três primeiros meses da terapia com AZA. Além disso, todos os pacientes foram alertados a retornarem prontamente à consulta em caso de dor faríngea, febre ou qualquer outro sinal de infecção. Leucopenia foi definida pela contagem total de leucócitos $<4.000$ células $/ \mathrm{mm}^{3}$ e leucopenia grave quando os mesmos estivessem abaixo de 2.000 células $/ \mathrm{mm}^{3}{ }^{13}$; convencionou-se como trombocitopenia a contagem de plaquetas $<130.000$ células $/ \mathrm{mm}^{3}$, neutropenia a contagem de neutrófilos $<1.000$ células $/ \mathrm{mm}^{3} \mathrm{e}$ linfopenia a contagem de linfócitos $<800$ células $/ \mathrm{mm}^{3}$, qualquer que fosse a ocasião da ocorrência de um destes eventos durante o tratamento com AZA.

Foram feitos ajustes da terapia durante o tratamento, objetivando uma dose ideal de AZA entre 2 e $3 \mathrm{mg} / \mathrm{kg} / \mathrm{dia}$ po. A dose inicial de AZA foi de $50 \mathrm{mg} / \mathrm{dia}$ com aumento gradual, dependendo da resposta clínica e dos ajustes necessários para prevenir leucopenia ou trombocitopenia ${ }^{13}$. Depois de duas semanas de terapia, desde que a leucometria total permanecesse acima de 10.000 células $/ \mathrm{mm}^{3}$, a dose era aumentada em $25 \mathrm{mg}$ a cada duas semanas, mantendo-se o monitoramento da contagem leucocitária até que esta atingisse níveis inferiores a 10.000 células $/ \mathrm{mm}^{3}$ ou até que a dose ideal baseada no peso corporal fosse atingida. Na vigência de efeitos adversos à AZA, várias abordagens foram adotadas, dependendo do julgamento clínico em cada caso individual. Isto poderia incluir: manutenção da mesma dose de AZA; redução transitória da dose e posterior aumento; redução permanente da dose; suspensão da droga com sua posterior reintrodução gradual; e retirada definitiva da AZA. Neste cenário, os pacientes tiveram sua evolução clínica rigorosamente acompanhada de perto pelo médico-assistente. Para fins comparativos, os pacientes foram divididos em grupos com e sem toxicidade à AZA.

A análise estatística foi realizada utilizando-se o programa SPSS I4.0 (SPSS, Chicago, IL, USA). Variáveis quantitativas foram expressas como mediana e variação ou como média \pm desvio padrão (DP), quando normalmente distribuídas. Estatística descritiva de todas as variáveis relevantes para os subgrupos foi calculada. A possível relação entre a ocorrência de leucopenia e náuseas/vômitos com as características demográficas e clínicas da população estudada foram analisadas utilizando-se o teste T de Student ou o teste do Qui quadrado, quando apropriados. Para comparações, o nível de significância estatística foi estabelecido em $p<0,05$.

\section{Resultados}

Foram incluídos prospectivamente 106 pacientes (45 homens e 61 mulheres) com DC em uso de AZA durante o período do estudo, sendo 86 (8I, I\%) pacientes brancos e 20 (I8,9\%) negros ou mulatos. A idade média do grupo foi de 36,3 anos (variação 12-68 anos), enquanto a duração da doença foi de 87,2 meses (variação 7-324 meses). As localizações mais comuns da doença foram ileocolônica $(39,6 \%)$ e íleo terminal (34\%) e o fenótipo predominante foi o fistulizante (45,3\%). A dose média de AZA foi de $1,9 \mathrm{mg} / \mathrm{kg} / \mathrm{dia}$ e a duração média do tratamento com a droga foi de 43,3 meses. As demais características dos pacientes encontram-se demonstradas na Tabela I. Globalmente, 56 (52,7\%) dos 106 pacientes apresentaram pelo menos um efeito adverso relacionado à terapia com AZA durante o seguimento. Efeitos adversos, muitas vezes associados entre si, levando à retirada definitiva da AZA ocorreram em 18 (I7\%) pacientes, sendo os principais: náuseas/vômitos incoercíveis $(n=9)$, dor abdominal $(n=5)$ e pancreatite aguda $(n=4)$, dentre outros. Além dos efeitos adversos citados, foram observadas outras complicações durante o tratamento com AZA: hepatite tóxica; náuseas/vômitos leves; mielossupressão; alterações nas provas de função hepática; complicações infecciosas; dentre outras (Tabela 2). Nenhum caso de neoplasia maligna foi registrado.

Pancreatite aguda foi observada em quatro $(3,8 \%)$ pacientes. $O$ tempo médio de surgimento da pancreatite foi de dois meses de uso da AZA (variação I-4 meses). Em todos os casos a AZA foi retirada definitivamente, e nenhum paciente desenvolveu pancreatite crônica ou foi a óbito devido à pancreatite. Hepatite tóxica foi observada em 


\begin{tabular}{|c|c|}
\hline Características & Valores' \\
\hline $\begin{array}{l}\text { Sexo } \\
\text { Homens } \\
\text { Mulheres }\end{array}$ & $\begin{array}{l}45(42,5) \\
61(57,5)\end{array}$ \\
\hline $\begin{array}{l}\text { Idade (anos) } \\
\text { Raça } \\
\text { Brancos } \\
\text { Negros }\end{array}$ & $\begin{array}{l}6,3 \pm 12,2 \\
86(81,1) \\
20(18,9)\end{array}$ \\
\hline Duração da doença (meses) & $87,2 \pm 63,7$ \\
\hline $\begin{array}{l}\text { Localização da doença } \\
\text { Ileoterminal } \\
\text { Cólon } \\
\text { lleocolônica } \\
\text { Trato gastrointestinal superior }\end{array}$ & $\begin{array}{c}36(34) \\
27(25,5) \\
42(39,6) \\
I(0,9)\end{array}$ \\
\hline $\begin{array}{l}\text { Fenótipo da doença } \\
\text { Não estenosante, não-fistulizante } \\
\text { Estenosante } \\
\text { Fistulizante }\end{array}$ & $\begin{array}{l}34(32,1) \\
24(22,6) \\
48(45,3)\end{array}$ \\
\hline Presença de comorbidades & $17(16)$ \\
\hline Cirurgias prévias & $38(35,8)$ \\
\hline Dose média de AZA (mg/kg/dia) & $1,9 \pm 0,7$ \\
\hline Tempo de uso da AZA (meses) & $43,3 \pm 23,8$ \\
\hline $\begin{array}{l}\text { Uso de outras medicações } \\
\text { Sulfassalazina } \\
\text { Mesalazina } \\
\text { Prednisona } \\
\text { Ciprofloxacino } \\
\text { Metronidazol }\end{array}$ & $\begin{array}{l}13(21) \\
16(25,8) \\
48(77,4) \\
2(3,2) \\
6(9,7)\end{array}$ \\
\hline
\end{tabular}

'Valores representam número de casos (porcentagem) ou média \pm desvio padrão

apenas um paciente e ocorreu dentro dos dois primeiros meses do tratamento. Os sintomas cederam com a suspensão definitiva da droga. Febre sem causa aparente ocorreu em 10 (9,4\%) pacientes, em média 30 meses após o início do tratamento com AZA (variação 5-69 meses). Em $40 \%$ dos casos de febre, a mesma dose de AZA foi mantida; em $40 \%$ dos casos, a AZA foi suspensa provisoriamente, permitindo 0 retorno posterior à mesma dose; e em $20 \%$ dos casos, a AZA foi retirada definitivamente (dois pacientes com febre logo no início do tratamento com AZA, sugerindo alergia). Todos os casos de febre cederam. Não foi observado nenhum caso de rash cutâneo. Observouse cinco casos (4,7\%) de dor abdominal sem pancreatite. A média de tempo de surgimento do sintoma foi de um mês (variaçãa l -3 meses) após o início da AZA. Em todos os casos a AZA foi suspensa definitivamente. Todos os pacientes evoluíram bem.

Náuseas e/ou vômitos foram observados em 29 (27,4\%) pacientes, em média 37 meses do uso da droga (variação I-90 meses). Em $52 \%$ destes pacientes foi possível manter a mesma dose de AZA; $31 \%$ dos casos tiveram a AZA retirada definitivamente devido à extrema intolerância a este medicamento; em 13,6\% dos casos, a AZA foi suspensa provisoriamente com posterior retorno à mesma dose; e

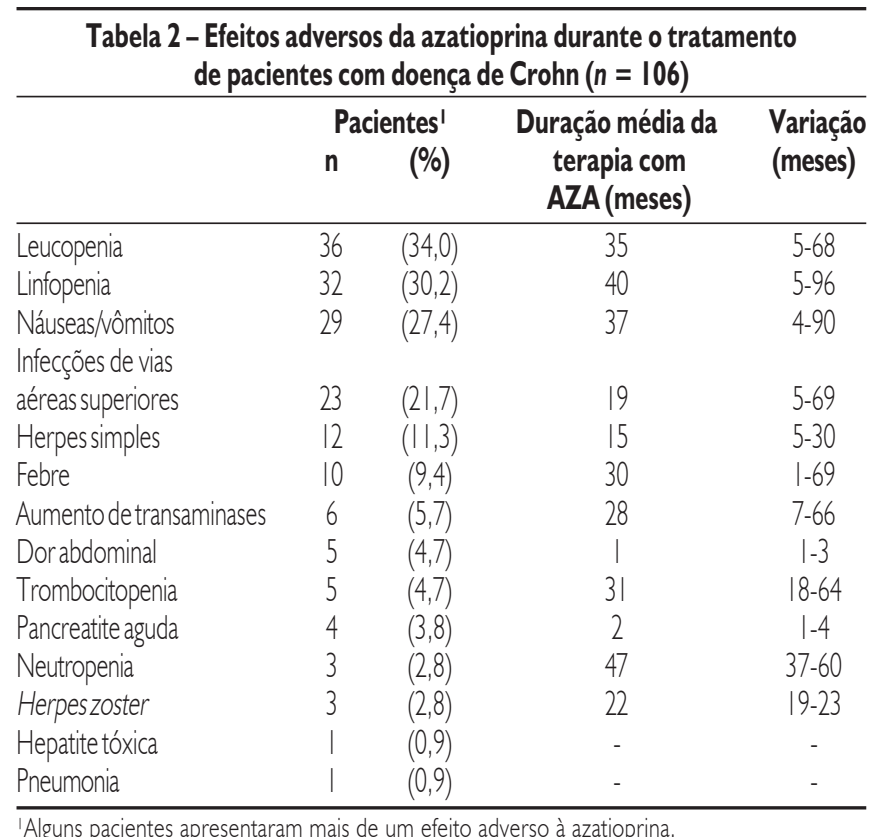

3,4\% dos pacientes necessitaram de redução permanente da dose de AZA. Em todos os casos os sintomas cederam. A dose e o tempo de uso de AZA foram similares nos subgrupos de pacientes com e sem náuseas/vômitos (I ,8 vs. I,9mg/kg/dia e 48,8 vs. 43,4 meses, respectivamente). Entretanto, pacientes negros e aqueles com comorbidades associadas à DC apresentaram significantemente mais náuseas e vômitos comparados a indivíduos brancos e sem comorbidades $(p=0,04)$. Pacientes negros apresentaram 2,7 vezes maior probabilidade de terem náuseas/vômitos do que os brancos ( $\mathrm{OR}=2,7$; IC: 0,98 - 7,44) e aqueles com comorbidades 2,9 mais chances de apresentarem este efeito adverso (OR=2,9; IC: 0,99 - 8,40).

Leucopenia foi registrada em 36 (34\%) pacientes, com uma média de tempo de surgimento de 35 meses (variação 5-68 meses) após o início da droga. Leucopenia grave ocorreu em cinco (4,7\%) casos. Do total de pacientes leucopênicos, 19,5\% foram manuseados com redução transitória da dose e posterior reajuste à dose anterior; $41,7 \%$ necessitaram da redução permanente da dose de AZA; em 33,3\% manteve-se a mesma dose de AZA; apenas dois (5,5\%) pacientes requereram a retirada definitiva da AZA. Não se observou septicemia nem óbitos nos pacientes leucopênicos. O tempo de uso de AZA foi significativamente maior no subgrupo de pacientes leucopênicos (54,2 meses) do que naqueles nãoleucopênicos (37,7 meses; $p=0,00$ I). Por outro lado, a dose média de AZA foi significativamente menor nos pacientes leucopênicos ( $1,6 \mathrm{mg} / \mathrm{kg} /$ dia) comparada aos não leucopênicos ( $2 \mathrm{mg} / \mathrm{kg} / \mathrm{dia} ; p=0,005)$. Todas as demais variáveis analisadas foram similares em ambos os subgrupos de pacientes (Tabela 3).

Três (2,8\%) pacientes tiveram neutropenia. A dose de AZA foi mantida ou transitoriamente reduzida em dois pacientes e a medicação definitivamente suspensa em um caso. Todos os pacientes evolúram bem. Observou-se linfopenia, sem conseqüências clínicas maiores, em 32 (30,2\%) pacientes. Em 75\% dos casos, a AZA foi mantida na mesma 


\begin{tabular}{|c|c|c|c|}
\hline Vaviónel & & & \\
\hline & Leucopenicos $(n-30)$ & & $r$ \\
\hline Dose de AZA (mg/kg/dia) & $1,6 \pm 0,7$ & $2,0 \pm 0,7$ & 0,005 \\
\hline Tempo de uso de AZA (meses) & $54,2 \pm 22,5$ & $40,0 \pm 21,5$ & 0,001 \\
\hline Idade (anos) & $35,7 \pm 11,2$ & $36,6 \pm 12,8$ & 0,70 \\
\hline Tempo de doença (meses) & $96,6 \pm 51,8$ & $82,3 \pm 68,8$ & 0,27 \\
\hline $\begin{array}{l}\text { Sexo } \\
\text { Masculino } \\
\text { Feminino }\end{array}$ & $\begin{array}{l}11 \\
25\end{array}$ & $\begin{array}{l}34 \\
36\end{array}$ & 0,07 \\
\hline $\begin{array}{l}\text { Raça } \\
\text { Brancos } \\
\text { Negros }\end{array}$ & $\begin{array}{c}31 \\
5\end{array}$ & $\begin{array}{l}55 \\
15\end{array}$ & 0,35 \\
\hline $\begin{array}{l}\text { Comorbidades } \\
\text { Sim } \\
\text { Não }\end{array}$ & $\begin{array}{c}7 \\
29\end{array}$ & $\begin{array}{l}10 \\
60\end{array}$ & 0,49 \\
\hline Uso de aminossalicilatos & 9 & 20 & 0,69 \\
\hline
\end{tabular}

dose; $22 \%$ dos pacientes requereram uma redução permanente da dose de AZA; e em apenas um paciente (3\%) a AZA foi retirada de forma permanente. Plaquetopenia foi observada em cinco $(4,7 \%)$ pacientes durante o tempo médio de uso da AZA de 3 I meses (variação 18-64 meses). Em três dos casos, a dose de AZA foi mantida; um paciente necessitou de redução permanente da dose de AZA e outro da suspensão definitiva da droga. Todos os efeitos adversos hematológicos foram resolvidos com as abordagens descritas acima.

Observou-se aumento das aminotransferases acima de duas vezes o limite superior da normalidade em seis (5,7\%) pacientes. O tempo médio de surgimento desta alteração foi de 28 meses (variação 7-66). Na maioria dos casos foi possível manter a mesma dose de AZA ou apenas a redução transitória da dose foi necessária para obter-se a normalização espontânea dos níveis séricos das aminotransferases. Em apenas um paciente, a AZA foi retirada definitivamente.

Apenas um paciente desenvolveu pneumonia, que ocorreu após 14 meses de uso de AZA. Este único caso evoluiu favoravelmente com o uso de antibiótico po, sendo a dose de AZA suspensa provisoriamente e, mais tarde, reintroduzida à dosagem anterior.

Herpes zoster ocorreu em três pacientes (2,8\%) durante o tempo médio de uso da droga de 22 meses (variação 19-23). Em dois destes pacientes, a AZA foi suspensa provisoriamente, com posterior retorno gradual à dose padrão. Em um paciente, a dose de AZA foi mantida na vigência da infecção herpética. Todos os pacientes se recuperaram sem seqüelas. Doze pacientes ( I I,3\%) apresentaram herpes simples localizado recidivante (I I nos lábios), em tempos variados do tratamento com AZA (média de 15 meses; variação 5-30 meses). Em todos os casos, a dose de AZA foi mantida e todos os pacientes evoluíram bem. Infecções recorrentes das vias aéreas superiores foram vistas em 23 pacientes $(21,7 \%)$, em ocasiões variadas do tratamento com AZA (média de 19,2 meses; variação 5-69 meses). Em apenas um caso a AZA foi transitoriamente suspensa. Os demais pacientes foram mantidos na mesma dose de AZA. Todos se recuperaram sem complicações.

\section{Discussão}

A AZA tem sido utilizada para o tratamento das DII por mais de 30 anos $^{14}$ e seu uso na DC tem sido avaliado em múltiplos ensaios randomizados-controlados desde $\left.197\right|^{15}$, que demonstram a sua eficácia no tratamento da DC. É uma droga geralmente bem tolerada, mas pode apresentar expressiva toxicidade' Os efeitos adversos associados à AZA podem ser categorizados como: não dose-dependentes (alérgicos ou idiossincrásicos) ou dose-dependentes. As reações alérgicas à AZA ou 6-MP ocorrem tipicamente dentro de duas a quatro semanas do início da terapia, e incluem febre, rash cutâneo, mialgias, dor abdominal, pancreatite e hepatite tóxica, e geralmente resolvem-se com a descontinuação da terapia ${ }^{16}$. Outros efeitos adversos gastrointestinais com AZA e 6-MP, não correlacionados com pancreatite, incluem náuseas e vômitos. Estes sintomas são usualmente leves e melhoram no decorrer do tempo ${ }^{7}$. Os efeitos adversos dosedependentes incluem mielossupressãa ${ }^{17} \mathrm{e}$ alterações nas provas de função hepática ${ }^{18}$.

A incidência total de efeitos adversos devido à AZA no presente estudo (52,7\%) foi maior que a relatada em estudos prévios (variação $27 \%$ a $41 \%)^{11,19}$, devido, pelo menos em parte, aos níveis considerados por nós como leucopenia (menor que 4.000 células $/ \mathrm{mm}^{3}$ ) contrastando com 3.000 células $/ \mathrm{mm}^{3}$ ou 3.500 células $/ \mathrm{mm}^{3}$ em outros estudos $^{11,19}$. Também consideramos como efeitos adversos os casos de neutropenia e linfopenia, que muitas vezes ocorreram na ausência de leucopenia global associada. Entretanto, eventos adversos significativos levando à retirada definitiva da AZA ocorreram em 18 (17\%) pacientes, similar ao observado em vários estudos (variação 10\% a 28\%) (16,20. $^{10}$. Náuseas/vômitos incoercíveis foram as causas mais freqüentes de interrupção da terapia, seguidas por dor abdominal e pancreatite. Náuseas/Vômitos precoces nos pacientes tratados com AZA ocorrem por reação alérgica ao anel imidazólico encontrado na AZA, sendo independentes da dose da droga ${ }^{21}$. Por isto, uma certa proporção de pacientes que experimentam uma reação alérgica à AZA poderão ser 
retratados com sucesso com a 6-MP21, já que esta não contém o anel imidazólico. A taxa de pancreatite observada foi similar à de outros estudos ${ }^{1,22}$, e todos os casos cederam após interrupção da droga. Em geral, pancreatite aguda deve-se a mecanismo de hipersensibilidade, independente da dose $\mathrm{e}^{22}$, ocorrendo precocemente no início da terapia $^{7}$ e levando à retirada definitiva da droga. Habitualmente a doença é leve e não deixa seqüelas` .

Globalmente, náuseas/vômitos (principalmente leves) foram observados em $27,4 \%$ dos pacientes, superior ao relatado em outros estudos I,20; porém, em 52\% destes pacientes os sintomas melhoraram com o tempo, sendo possível manter a mesma dose de AZA (Tabela 2). É interessante notar que os pacientes negros apresentaram significantemente mais náuseas/vômitos do que os brancos $(p=0,04)$. A tiopurina metiltransferase (TPMT) é uma das enzimas mais importantes no metabolismo da AZA/6-MP7. Existem variações étnicas nos alelos mutantes da TPMT ${ }^{23}$ e mais de 20 variantes alélicos associados com redução da atividade da TPMT têm sido descritos ${ }^{24,25}$. As diferenças herdadas na TPMT são responsáveis pela maior parte da variabilidade na resposta à droga entre os indivíduos ${ }^{7}$. Como o polimorfismo genético conhecido da TPMT tem sido melhor avaliado em populações caucasianas ${ }^{24}$, podemos hipotetizar que diferenças no perfil metabólico da AZA nos negros podem ter sido responsáveis pela maior ocorrência de náuseas/vômitos nestes pacientes do que nos brancos. Em adição, pacientes com outras comorbidades também apresentaram significativamente maior incidência de náuseas/vômitos do que aqueles sem comorbidades $(p=0,04)$. É admissível supor que a presença de comorbidades, quer seja por elas mesmas, pelo uso concomitante de outras medicações ou por mudanças no perfil metabólico da AZA, tenha favorecido a maior ocorrência de náuseas/ vômitos nesse subgrupo de pacientes. Esta teoria, no entanto, permanece em caráter especulativo, merecendo investigações posteriores.

Mielossupressão foi o evento adverso mais freqüente (Tabela 2), tendo ocorrido em qualquer época do tratamento com AZA, similar ao observado em outros estudos ${ }^{11,13}$. Sua manifestação incluiu: leucopenia (34\%), linfopenia (30,2\%), trombocitopenia $(4,7 \%)$ ou neutropenia (2,8\%), ocorrendo associadas ou não. Leucopenia grave ocorreu em apenas cinco casos, o que provavelmente justifica não termos observado infecções graves, septicemia e nem óbito nesta casuística; os demais efeitos hematológicos cursaram sem conseqüências clínicas maiores. Por serem dose-dependentes, os efeitos adversos hematológicos foram eficazmente manuseados com redução transitória ou manutenção da mesma dose na maioria dos casos e, menos comumente, com a diminuição permanente da dose. $\mathrm{O}$ tempo de uso de AZA foi significativamente maior no subgrupo de pacientes leucopênicos (54,2 meses) do que naqueles nãoleucopênicos (37,7 meses) (Tabela 3). Este achado pode ser parcialmente explicado, conforme relatado por outros autores ${ }^{13}$, pelo fato da mielossupressão ocorrer a qualquer tempo da terapia com AZA, tão longo quanto $\mid \mathrm{I}$ anos ${ }^{13}$. Conseqüentemente, torna-se necessária uma contínua monitorização da contagem das células sangüíneas em intervalos regulares, durante todo o período da terapia. Por outro lado, a dose média de AZA foi significativamente menor nos pacientes leucopênicos (1,6 mg/kg/dia),comparado aos nãoleucopênicos (2 mg/kg/dia). Sabe-se que os metabólitos ativos da
AZA, principalmente a 6-tioguanina (6-TGN), são os primariamente responsáveis pela mielotoxicidade da terapia com $A Z A^{7}$. Estudos têm demonstrado níveis significativamente mais altos de 6-TGN nos eritrócitos dos pacientes que desenvolvem leucopenia comparados com aqueles sem leucopenia ${ }^{26}$. Níveis mais elevados de 6-TGN correlacionam-se com deficiência da enzima TPMT e pacientes com atividade muito baixa ou ausente desta enzima (geneticamente determinada) podem experimentar grave mielossupressãa 27 . É possível, portanto, que os pacientes leucopênicos do nosso estudo, apesar de terem recebido doses menores de AZA, tivessem alcançado concentrações mais elevadas de 6-TGN eritrocitária do que os nãoleucopênicos, por diferenças genéticas intrínsecas. A leucopenia, entretanto, pode ocorrer com níveis normais de 6-TGN7. Portanto, a dosagem dos níveis de 6-TGN não deve substituir a monitorização laboratorial de rotina, e não é recomendada, até o momento, para reduzir a ocorrência de mielossupressão ${ }^{7}$.

Elevação discreta das aminotransferases séricas foi observada em $5,7 \%$ dos casos, em conformidade com outros estudos, que mostram uma elevação dose-dependente das aminotransferases em $1 \%$ a $10 \%$ dos pacientes ${ }^{11,28}$. No presente estudo, obteve-se a normalização espontânea das aminotransferases, apesar de termos mantido a mesma dose de AZA na maioria dos casos. Este fato já foi previamente observado por outros autores ${ }^{29}$. Alguns estudos têm relatado hepatite clínica droga-induzida, confirmada por resposta à suspensão da droga ${ }^{16}$. Observamos apenas um caso, cujos sintomas cederam após a retirada da AZA.

A terapia com AZA está associada a um aumento do risco de infecções ${ }^{7}$, principalmente se os corticosteróides fazem parte do esquema terapêutico ${ }^{28}$. Apenas um paciente desenvolveu pneumonia no presente estudo, que evoluiu favoravelmente com antibióticos orais e temporária descontinuação da terapia com AZA. Infecções recorrentes das vias aéreas superiores foram observadas em 21,7\% dos casos, superior ao relatado em outros estudos ${ }^{11,19}$. Sejam ou não devidas ao uso de AZA, todos os pacientes se recuperaram sem complicações, sendo a droga suspensa provisoriamente em apenas um caso. O desenvolvimento de Herpes zoster foi observado em $2,8 \%$ dos pacientes, similar a relatos anteriores ${ }^{11,19}$. Parece que 0 risco de desenvolver Herpes zoster em pacientes usando AZA ou 6-MP para o tratamento das Dlls é um pouco maior do que na população com Dll em geral e a maioria dos casos tem um curso benigno ${ }^{29}$. Observamos também II,3\% de casos de herpes simples localizado recidivante, o que não difere da incidência anual dos herpes na população geral (12\%)". Em todos estes casos, a dose de AZA foi mantida. Como previamente comentado, leucopenia grave foi rara em nossa casuística. Além disso, nossa rotina incluiu supervisão clínica e determinação periódica da leucometria em todos os pacientes em uso de AZA, visando possíveis reajustes na dosagem da droga. Provavelmente, estes fatos justificam não termos observado infecções virais ou bacterianas graves durante o tratamento prolongado com AZA.

Embora não tenhamos registrado nenhuma malignidade, esta tem sido relatada como sendo de 3\% a 4\% em pacientes com DII tratados com os análogos da purina ${ }^{16,30}$. Esta freqüência não parece ser maior do que aquela da população com DII não recebendo estes agentes ${ }^{30}$. 
Entretanto, é ainda controverso se o uso das tiopurinas está associado a um risco aumentado de linfomas ${ }^{31}$. Alguns estudos, baseados em dados populacionais, relatam um aumento discreto do risco de linfoma em pacientes com DII tratados com estes agentes ${ }^{30}$. Em pacientes com DC tratados com AZA ou 6-MP, o desenvolvimento de linfoma parece estar associado com infecção pelo vírus Epstein-Barr ${ }^{32}$. Entretanto, uma análise de decisão concluiu que os benefícios da terapia com AZA superam o possível risco de linfoma nas DI ${ }^{33}$.

\section{Conclusão}

Nossos dados demonstram ser a azatioprina uma droga relativamente segura no tratamento da DC. Embora a ocorrência de efeitos adversos tenha sido comum (52,7\%), na maioria dos pacientes eles foram leves e autolimitados, mesmo sendo mantida a mesma dose de AZA ou apenas reduzindo-a transitoriamente. Retirada definitiva da AZA devido a efeitos adversos ocorreu em 17\% dos casos, geralmente em virtude de reações de hipersensibilidade. Supervisão clínica periódica e monitorização sérica, particularmente da leucometria, são mandatórias durante todo o tratamento. Como todas as terapias, os benefícios devem ser pesados contra as potenciais toxicidades.

\section{Conflito de interesse: não há}

\section{SUMMARY}

\section{Azathioprine toxicity in Crohn's disease: incidence, APPROACH AND COURSE}

OBJECTIVE. Azathioprine (AZA) is frequently used in Crohn's disease (CD) therapy. This paperaimed to evaluate the frequency, evolution and management of $A Z A$ side effects in $C D$ patients.

METHODS. One hundred and six CD patients under AZA therapywere evaluated prospectively from January 2002 to December 2006. Clinical and demographic data were recorded, together with a monthly laboratory control of hematological or other adverse reactions by means of clinical evaluation. Comparison was carried out between groups with and without side effects.

RESULTS. At least one adverse reaction was found in 56 (52.7\%) of the patients studied and required a transient drug reduction; 18 (17\%) had to definitely stop use of AZA, often because of hypersensitivity reactions. Nausea, vomit, although slight, occurred in 29 (27.4\%). The black race and those with co-morbidities had more gastric intolerance than Caucasians and those without other associated disease $(p=0.04)$. Leucopoenia was the more frequent side effect observed, occurring in 36 (34\%). The period of AZA use was longer for patients with leucopoenia than for those without $(p=0.001)$, while the mean dose of $A Z A$ was lower for those with leucopoenia when compared to non-leucopoenics $(p=0.005)$. No serious infections, malignancy or death was noticed as a consequence of AZA use.

CONCLUSION. In this study use of AZA in therapy for Crohn's disease disclosed that the drug is satisfactorily safe as long as periodical clinical and laboratory supervision is carried out during treatment. [Rev Assoc Med Bras 2008; 54(5): 415-2I]

KEY WORDS: Crohn's disease. Azathioprine. Toxicity.

\section{REFERÊNCIAS}

I. Lennard-Jones E. Classification of inflammatory bowel disease. Scand J Gastroenterol. 1989;24:2-6.

2. Gaburri PD, Castro LEVV, Ferreira JOD, Lopes MHM, Ribeiro AMR, Alves RA, et al. Epidemiologia, aspectos clínicos e evolutivos da Doença de Crohn: um estudo de 60 casos. Arq Gastroenterol. 1998;4:240-6

3. Fellows IW, Freeman IG, Holmes GK. Crohn's disease in the city of Derby, 1951-85. Gut. 1990;31:1262-5.

4. Shivananda S, Lennard-Jones J, Logan R, Fear N, Price A, Carpenter L, et al. The EC-IBD Study Group. Incidence of inflammatory bowel disease across Europe: is there a difference between north and south? Results of the European collaborative study on inflammatory bowel disease (ECIBD). Gut. 1996;39:690-7.

5. Souza MHLP, Troncon LEA, Rodrigues CM, Viana CFG, Onofre PHC, Monteiro RA, et al. Evolução da ocorrência (1980-1999) da doença de Crohn e da retocolite ulcerativa idiopática e análise das suas características clínicas em um hospital universitário do sudeste do Brasil. Arq Gastroenterol. 2002;39:98- 105.

6. Griffin MG, Miner PB. Conventional drug therapy in inflammatory bowel disease. Gastroenterol Clin North Am. 1995;24:509-12.

7. Su C, Lichtenstein GR. Treatment of inflammatory bowel disease with azathioprine and 6-mercaptopurine. Gastroenterol Clin North Am. 2004;33:209-34.

8. Sandborn W, Sutherland L, Pearson D, May G, Modogliani R, Prantera G. Azathioprine or 6-mercaptopurine for inducing remission of Crohn's disease. Cochrane Database Syst Rev. 2000(CD 000545).

9. Pearson DC, May GR, et al. Azathioprine for maintenance of remission in Crohn's disease. Cochrane Database Syst Rev. 2003;3.

10. Siegel C, Olenec C, Sands BE. Review article: practical management of inflammatory bowel disease in patients taking immunomodulators. Aliment Pharmacol Ther.2005;22: I - 16.

I I. Chebli JMF, Gaburri PD, Souza AFM, Pinto ALT, Chebli LA, Felga GEG, et al. Long-term results with azathioprine therapy in patients with corticosteroid-dependent Crohn's disease: Open-label prospective study. J Gastroenterol Hepatol. 2007;22:268-74.

12. Gasche C, Scholmerich J, Brynskov J, D' Haens G, Hanauer SB, Irvine EJ, et al. A simple classification of Crohn's disease: report of the Working Party for the World Congresses of Gastroenterology, Vienna. Inflamm Bowel Dis. 2000;6:8- I5.

13. Connell WR, Kamm MA, Ritchie JK, Lennard-Jones JE. Bone marrow toxicity caused by azathioprine in inflammatory bowel disease: 27 years of experience. Gut. 1993;34: 1081-5.

14. Brooke BN, Hoffmann DC, Swarbrick ET. Azathioprine for Crohn's disease. Lancet. 1969;2:612-4.

15. Pearson DC, May GR, Fick GH. Azathioprine and 6-mercaptopurine in Crohn's disease. A meta-analysis. Ann Intern Med. 1995; I 23: I 32.

16. Present DH, Meltzer SJ, Krumholz MP, Wolke A, Korelitz BI. 6Mercaptopurine in the management of inflammatory bowel disease: short- and long-term toxicity. Ann Intern Med. 1989; I I 1:64 I-9.

17. Colombel JF, Ferrari N, Debuysere H, Marteau P, Gendre JP, Bonaz B, et al. Genotypic analysis of thiopurine S-methyltransferase in patients with Crohn's disease and severe myelosuppression during azathioprine therapy. Gastroenterology. 2000; I 1 8: 1 025-30.

18. Bouhnik Y, Lemann M, Mary JY, Scemama G, Tai R, Matuchansky C, et al. Long-term follow-up of patients with Crohn's disease treated with azathioprine or 6-mercaptopurine. Lancet. 1996;347:215-9.

19. Warman JI, Korelitz BI, Fleisher MR, Janardhanam R. Cumulative experience with short- and long-term toxicity to 6-mercaptopurine in the treatment of Crohn's disease and ulcerative colitis. J Clin Gastroenterolol. 2003:37:220-5.

20. Winter JW, Gaffney D, Shapiro D, Spooner RJ, Marinakis AM, Sanderson $J D$, et al. Assessment of thiopurine methyltransferase enzyme activity is superior to genotype in predicting myelosupression following azathioprine therapy in patients with inflammatory bowel disease. Aliment Pharmacol Ther. 2007;25: 1069-77. 
21. McGovern DP, Travis SP, Duley T, Shobowale-Bakre el M, Dalton HR. Azathioprine intolerance in patients with IBD may be imidazole-related and is independent of TPMT activity. Gastroenterology. 2002; I 22:838-89.

22. Present DH, Korelitz BI, Wisch N, Glass JL, Sachar DB, Pasternack BS. Treatment of Crohn's with 6-mercaptopurine. N Engl J Med. 1980;302:98|-7.

23. Otterness D, Szumlanski C, Lennard L, Klemetsdal B, Aarbakke J, Park$\mathrm{Hah} \mathrm{JO}$, et al. Human thiopurine methyltransferase pharmacogenetics: gene sequence polymorphisms. Clin Pharmacol Ther. 1997;62:60-73.

24. Schaeffeler E, Fischer C, Brockmeier D, Wernet D, Moerike K, Eichelbaum M. Comprehensive analysis of thiopurine S-methyltransferase phenotype-genotype correlation in a large population of GermanCaucasians and identification of novel TPMT variants. Pharmacogenetics. 2004; 14:407-17

25. Sasaki T, Goto E, Konno Y, Hiratsuka M, Mizugaki M. Three novel single nucleotide polymorphisms of the human thiopurine S-methyltransferase gene in Japanese individuals. Drug Metab Pharmacokinet. 2006;21:332-6.

26. Dubinsky MC, Lamothe S, Yang HY, Targan SR, Sinnett D, Theoret $Y$, et al. Pharmacogenomics and metabolite measurement for 6mercaptopurine therapy in inflammatory bowel disease. Gastroenterology. 2000; 1 18:705- 13.

27. Lennard L, Gibson BES, Nicole T, Lilleyman JS. Congenital thiopurine methyltransferase deficiency and 6-mercaptopurine toxicity during treatment for acute lymphoblastic leukemia. Arch Dis Child. 1993;69:577-9.
28. Bonis PA, MacDermott RP. Immunomodulator therapy in Crohn's disease. In: UpToDate. Rose BD, editor. Waltham: UpToDate; 2006.

29. Dubinsky MC, Yang H. Azathioprine, 6-mercaptopurine in inflammatory bowel disease: pharmacology, efficacy, and safety. Clin Gastroenterol Hepatol. 2004;2:73 I-43.

30. Connell WR, Kamm MA, Dickson M, Balkwill AM, Ritchie JK, LennardJones JE. Long-term neoplasia risk after azathioprine treatment in inflammatory bowel disease. Lancet. 1994;343: | 249-52.

31. Lewis JD, Bilker WB, Brensinger C, Deren JJ, Vaughn DJ, Strom BL. Inflammatory bowel disease is not associated with an increased risk of lymphoma. Gastroenterology. 200 I; I 21:1080-7.

32. Dayharsh GA, Loftus EV Jr, Sandborn WJ, Tremaine WJ, Zinsmeister AR, Witzig TE, et al. Epstein-Barr virus-positive lymphoma in patients with inflammatory bowel disease treated with azathioprine or 6mercaptopurine. Gastroenterology. 2002; 122:72-7.

33. Lewis JD, Schwartz JS, Lichtenstein GR. Azathioprine for maintenance of remission in Crohn's disease: benefits outweigh the risk of lymphoma. Gastroenterology. 2000; | | 8: 10 18-24.

Artigo recebido: 18/08/07

Aceito para publicação: 10/04/08 